\title{
TTR
}

Traduction, terminologie, rédaction

\section{De Bellefeuille sous la plume de D. G. Jones}

\section{Patricia Godbout}

Volume 28, numéro 1-2, 1er semestre-2e semestre 2015

La traduction littéraire et le Canada

Literary translation and Canada

URI : https://id.erudit.org/iderudit/1041654ar

DOI : https://doi.org/10.7202/1041654ar

Aller au sommaire du numéro

\section{Éditeur(s)}

Association canadienne de traductologie

ISSN

0835-8443 (imprimé)

1708-2188 (numérique)

Découvrir la revue

Citer cet article

Godbout, P. (2015). De Bellefeuille sous la plume de D. G. Jones. TTR, 28(1-2), 139-151. https://doi.org/10.7202/1041654ar

\section{Résumé de l'article}

La traduction, par D. G. Jones, du recueil de poèmes Catégoriques un deux et trois (1986) de Normand de Bellefeuille est examinée ici à la lumière des réflexions que livre notamment Arno Renken sur le rejet traditionnel de la traduction au nom de sa non-identité avec le texte original. Plusieurs choix de traduction faits par D. G. Jones dans Categorics one two \& three (1992) sont étudiés afin de voir les effets combinés que ces diverses transpositions produisent sur la lecture du recueil en langue anglaise. 


\section{De Bellefeuille sous la plume de D. G. Jones}

\section{Patricia Godbout}

Université de Sherbrooke

\section{Résumé}

La traduction, par D. G. Jones, du recueil de poèmes Catégoriques un deux et trois (1986) de Normand de Bellefeuille est examinée ici à la lumière des réflexions que livre notamment Arno Renken sur le rejet traditionnel de la traduction au nom de sa non-identité avec le texte original. Plusieurs choix de traduction faits par D. G. Jones dans Categorics one two E three (1992) sont étudiés afin de voir les effets combinés que ces diverses transpositions produisent sur la lecture du recueil en langue anglaise.

Mots-clés: D. G. Jones, poésie, Normand de Bellefeuille, transposition, lecture

\section{Abstract}

The English translation, by D. G. Jones, of Catégoriques un deux et trois (1986), a book of poems by Normand de Bellefeuille, is examined through the reflections proposed by Arno Renken, among others, on the question of the common rejection of a translation on the grounds of its non-identity with the original. Many translation choices made by Jones in Categorics one two E three (1992) are analyzed in order to bring into light the combined effects these various transpositions produce on the reader of the English version.

Keywords: D. G. Jones, poetry, Normand de Bellefeuille, transposition, reading

En recevant, en 2009, le prix Raymond Aron pour les traductions franco-allemandes en sciences humaines et sociales, le traducteur Arno Renken a rappelé que la réaction la plus fréquente à l'égard de la traduction consiste à reprocher à celle-ci de ne pas être l'original ${ }^{1}$. Selon lui, il est grand temps d'abandonner cette perspective normative et d'interroger ce rejet de la traduction au nom d'une non-identité.

1. On peut lire le texte de ce discours d'Arno Renken, «Traduction et étrangeté», à l'adresse suivante: http://www.riponne.ch/textes/200912_AR_ prixAron.htm. 
Pour Renken, qui reprend cette argumentation dans Babel heureuse, il importe de ne pas oublier qu'il «n'y a pas de lieu sans langue pour parler des langues et [qu'] il n'y a pas de langue universelle qui englobe les autres» (2012, p. 25). Cela a, en matière de traduction, deux conséquences: «[t] out discours sur la traduction, comme tout discours sur les langues, se fait dans une langue» (ibid.). Ainsi, affirme-t-il,

même les catégories morales ou méthodologiques par lesquelles on enclôt la traduction dans sa fonction de médiation transparente (fidélité, exactitude, justesse, etc.) participent elles aussi d'une langue particulière et résistent ainsi à leur manière au passage, fidèle, exact et mesurable, d'une langue à l'autre. (Renken, 2009, n.p.)

Ensuite, cela nous amène à reconnaître que «la traduction est une figure par laquelle les langues sont étrangères, parce que quelque chose échappe entre les textes qui ne sera jamais pleinement formulable et récupérable par aucune langue ni aucun discours" (ibid.). Renken reprend ensuite la définition qu'a proposée de la traduction le poète Oskar Pastior, pour qui celle-ci est «un texte et un texte» $(2012$, p. 29), un texte original et une traduction, côte à côte.

À la lecture comparative des versions originale française et anglaise d'un recueil de poèmes de Normand de Bellefeuille, il m’a semblé que cette idée de juxtaposition, éloignée d'un certain idéal de remplacement, était susceptible d'éclairer la démarche traductive adoptée en loccurrence par D. G. Jones. Dans cette perspective, le regard porté sur sa traduction anglaise du recueil de poèmes de Normand de Bellefeuille, Catégoriques un deux et trois, pourrait ainsi s'intituler: "Un texte, une traduction et une lecture critique». Le mouvement ternaire constitutif de cette «catégorique» inclurait donc la traduction et le regard posé sur celle-ci. À cet égard, Renken précise que «toute critique de traduction est par nécessité linguistiquement partisane et ellemême tributaire d'un geste de traduction» (2009, n.p.). Ainsi, le fait de traiter en français de cette traduction anglaise de Jones est «linguistiquement partisan», en ce sens que c'est dans cette langue, dans les mots de cette langue et non dans un lieu hors-langue, qu'elle est examinée. Les «catégories» auxquelles se réfère Renken - fidélité, exactitude, justesse - à l'aune desquelles les traductions 
sont généralement évaluées seront ici mises de côté pour faire place à un autre type d'examen des «catégoriques» chez de Bellefeuille et des «categorics» chez Jones, examen qui cherchera à déterminer de quelle façon la traduction de Jones accompagne les poèmes en langue française plutôt qu'elle ne chercherait à se substituer à eux. On pourrait faire un certain rapprochement avec le concept de non-traduction proposé par Jacques Brault, dans ses Poèmes des quatre côtés, dans la mesure où c'est la manière qu'a le traducteur de pénétrer l'espace d'écriture du texte original qui est ici interrogée.

Mais d'abord, il n'est sans doute pas inutile de rappeler qui est D. G. Jones, traducteur de ce recueil de Normand de Bellefeuille qui parut en 1992 sous le titre Categorics one two Ev three (Toronto, Coach House Press) ${ }^{2}$. Originaire de l'Ontario, Doug Jones, qui est décédé le 6 mars 2016, a vécu à North Hatley, dans les Cantons de l'Est, pendant un demi-siècle. Il a été professeur de littérature canadienne comparée à l'Université de Sherbrooke de 1963 à 1994. Il était lui-même poète, auteur d'une dizaine de recueils. Il a été lauréat de deux prix littéraires du Gouverneur général, l'un remporté en 1977 pour son recueil Under the Thunder the Flowers Light Up the Earth et le second en 1993 pour sa traduction du recueil de de Bellefeuille.

Jones est en outre l'auteur d'un important ouvrage critique, Butterfly on Rock: A Study of Themes and Images in Canadian Literature (Toronto University Press, 1970). E. D. Blodgett écrit au sujet de ce livre qu'on y trouve une analyse de la littérature canadienne qui s'éloigne sensiblement de la vision canonique exprimée par le réputé critique Northrop Frye. Pour Frye, la «mentalité de garnison» propre aux Canadiens aurait amené ceuxci à percevoir la nature ou le milieu environnant comme un Autre aliénant et aurait fait naître chez eux un désir de domination de cet espace ${ }^{3}$. Jones se préoccupe quant à lui, dans son étude, de l'écriture canadienne du sujet comme espace de lutte existentielle. «Le conflit trouve à s'exprimer en lien avec la relation qu'entretient le sujet avec la nature», explique Blodgett (2012a, p. 16). Ce dernier estime en outre que le regard autre que porte Jones sur la littérature

2. Notons que l'emploi, en anglais, de l'esperluette donne à ce titre un côté un tant soit peu plus institutionnel.

3. Frye a exposé cette idée dans sa conclusion à l'ouvrage Literary History of Canada publié en 1965 sous la direction de Carl F. Klinck. 
canadienne, lequel était inusité à son époque, émanait en partie de sa connaissance de la culture du Québec et de sa compréhension du rapport différent que cette dernière entretient avec le temps, dont elle se préoccuperait davantage que la culture canadienneanglaise, celle-ci étant surtout subjuguée par l'espace (ibid., p. 15).

Jones a également fait connaître par l'entremise de ses traductions de grands poètes québécois comme Paul-Marie Lapointe et Gaston Miron aux communautés canadienne-anglaise et internationale. Il a fondé en 1969 la revue de traduction poétique Ellipse, périodique auquel il a collaboré pendant une trentaine d'années. C'est d'ailleurs au départ afin de faire lire des poésies québécoises à ses étudiants dans ses séminaires de littérature canadienne comparée que Jones s'est mis à en traduire.

Dans un article intitulé "Grounds For Translation", Jones note la difficulté de traduire le poème «Soir d'hiver» de Nelligan. Rejetant néanmoins l'idée de la nature intraduisible de la poésie, il revendique la liberté de "mal traduire», de jouer avec la matière donnée, de l'inverser, la transformer, la recréer, car c'est ce qui nourrit la poésie, l'écriture (Jones, 1977, p. 86). À ses yeux, donc, le poème et sa traduction sont "un texte et un texte», ou, comme le dit Normand de Bellefeuille, «ça ne revient jamais vraiment au Même» (1986, p. 22).

Le désir adamique de possession de l'espace qui s'exprime dans la poésie canadienne-anglaise a été souligné de nouveau par Blodgett dans un article paru dans le numéro de la revue Études françaises faisant suite au colloque sur « Saint-Denys Garneau dans le grand contexte», qui s'était tenu à Québec en avril 2012 pour souligner le centenaire de la naissance du poète. Blodgett, qui se penche en particulier sur les traductions anglaises de Garneau par John Glassco, explique de façon très convaincante que la difficulté que pose la poésie de Saint-Denys Garneau pour ses lecteurs anglophones ne se situe pas sur le plan proprement linguistique mais réside plutôt dans leur imaginaire (2012b, p. 112). Il montre que la poésie anglo-canadienne, plus narrative, est davantage préoccupée par la nécessité de nommer, tandis que la poésie québécoise se fait plus intérieure (ibid., p. 119). Qui connaît les vers de Doug Jones a pu constater qu'ils sont souvent imprégnés d'une ambiance méditative. Cela le rendrait-il mieux apte à traduire les poèmes de de Bellefeuille? 


\section{Un manuscrit primé}

Publié il y a un peu plus d'un quart de siècle, Catégoriques un deux et trois a reçu le "Grand Prix de Poésie de la Fondation des Forges» sur manuscrit. L'auteur avait jusque-là publié aux Herbes Rouges et à la Nouvelle Barre du Jour. Le jury était composé de Paul Chamberland, de Joseph Bonenfant et de Ginette Michaud. Cette dernière était à l'époque une jeune professeure de lettres à l'Université de Montréal. Paul Chamberland était bien connu comme écrivain et éditeur depuis les années soixante. Quant au regretté Joseph Bonenfant (1934-2000), il était alors professeur de littérature et de création littéraire à l'Université de Sherbrooke. Il s'agit donc d'un jury très qualifié et le manuscrit retenu valait indéniablement d'être publié.

Le recueil se divise en trois parties, composée chacune de vingt poèmes en prose. Ces trois parties sont précédées d'un texte intitulé «Catégoriques». La citation de Cioran mise en exergue («à quoi bon écrire pour dire exactement ce qu'on avait à dire») donne le ton, le rappel de la non-coïncidence de l'écriture avec elle-même étant en effet au cœur de la poétique de ce recueil. Comme premier décalage, ou écart, il y a celui qu'opère le mot "catégoriques» du titre - qui est aussi le titre du poème liminaire. On se demande en effet si on doit le lire comme un adjectif ou un substantif. Le mot «catégorie», qui nous vient à l'esprit, est d'ailleurs présent, au pluriel, dans ce premier poème. On remarquera, en examinant la traduction de Jones, qu'il ne se sent pas tenu de rendre toujours le mot "catégories" par «categories ", et ce, même s'il s'agit d'un terme clé du recueil. Par exemple, là où l'on trouve, dans le poème "À petits coups de chaussons légers", le segment «Le haut et le bas ne sont que des catégories de la limite» (de Bellefeuille, 1986, p. 46), celui-ci est rendu en anglais par «High and low are merely limit terms» (de Bellefeuille, 1992, p. 44).

Pour revenir au poème liminaire, sa syntaxe voudrait amener le lecteur à s'immiscer pour ainsi dire dans une discussion en cours. C'est comme si on lui demandait d'entrée de jeu de saisir de quoi il est question: "Mais il y en a aussi de voix, de gestes, de rythmes " (de Bellefeuille, 1986, p. 9) - éléments qui sont, notons-le, autant de catégories. Dans la traduction, l'entrée en matière est un peu différente, un peu plus explicative, un peu moins répétitive. « But there is also the question of voice, the gestures, the rhythms» (de 
Bellefeuille, 1992, n.p.). On trouve par ailleurs dans ce premier texte le mot «trois»: "trois ombres emmêlées» (celles d'Aristote, de Kant et de Nietzsche).

Jones ne peut pas, évidemment, rendre chaque jeu sur les signifiants de manière à créer un effet identique dans le poème traduit en anglais. Par exemple, dans le poème "Indifférence, murmure", le syntagme "une toux et sa quinte» (de Bellefeuille, 1986, p. 15) contient un évident jeu de mots puisqu'il est question de musique dans ce premier tiers du recueil. Jones ne retient quant à lui ni l'équivalent figé que donne le dictionnaire bilingue pour «quinte de toux» («coughing fit») et il ne choisit pas non plus d'intégrer à ce syntagme le terme utilisé en musique pour rendre «quinte» ( fifth»), ce qui aurait pu donner «coughing fifth". Il traduit plutôt ce passage par "a cough and its croup" (de Bellefeuille, 1992, p. 13): en plus de créer une allitération, il conserve ainsi le sens médical de l'expression.

Le mouvement ternaire induit par le recueil amène la troisième voix critique à interpréter en fonction des exigences de la traduction certains des énoncés poétiques que contient ce recueil. C'est le cas d'un élément rhétorique central de ces poèmes, la répétition, à propos de laquelle diverses assertions sont faites par le poète. L'une d'elles se lit comme suit: "Nous sommes si peu préparés à la répétition" (de Bellefeuille, 1986, p. 15). Si l'on rapproche une telle affirmation des remarques que font Vinay et Darbelnet sur les différences stylistiques entre le français et l'anglais, on observe que celle-ci semble plus «vraie» en français qu'en anglais. En effet, ces deux linguistes font observer que «l'anglais ne craint pas les répétitions, au contraire» (Vinay et Darbelnet, 1977, p. 252), le français s'en accommodant en général beaucoup moins bien. Ainsi, l'assertion «Nous sommes si peu préparés à la répétition » aurait un caractère d'évidence moins net (évidence induite, évidemment, en français par la parole poétique) dans la langue de la traduction. La figure de la répétition est d'ailleurs accentuée çà et là dans la version anglaise. Ainsi, le segment «Ce sont des choses qu'on dit les petits soirs de deuil et de fécondité»(de Bellefeuille, 1986, p. 37), tiré du poème "Quatre fois sur des airs lents », est rendu par Jones de manière délibérément redondante: "These are the things one says evenings of mourning and rejoicing, of mortality and fecundity » (de Bellefeuille, 1992, p. 35). 
Il est certain que des phrases traitant du «bonheur de la répétition» (de Bellefeuille, 1986, p. 22), sans parler de la répétition elle-même pratiquée de manière soutenue dans le recueil, font indubitablement partie du «message» que le lecteur capte dans ces poèmes. Cela l'oblige à examiner quel effet esthétique cette figure produit sur lui. Dans le poème "Ces musiques-là dans les champs d'échalottes $[s i c]$ ", il est question d' "une mémoire sans fin de la fête première » qui condamne à la répétition (ibid., p. 26) : la répétition serait donc liée à l'origine, au temps, dans lequel on cherche à s'inscrire, quête que la réflexion sur la danse de la deuxième partie du recueil contribuera cependant à obscurcir. On se prend à faire des liens entre ces préoccupations poétiques et les remarques de Jones et Blodgett sur le rapport différent à l'espace et au temps tel qu'il s'exprime dans la poésie anglo-canadienne et québécoise. On observera par exemple que la manière de caractériser le son, décrit - dans le poème «Mortel, j'avais une voix» - comme étant le «commentaire irréductible et net de la durée» (ibid., p. 21), inscrit celui-ci dans le temps.

Le fait que certains mots des poèmes de Normand de Bellefeuille ont le potentiel de faire écho à d'autres mots ou concepts peut se trouver répercuté de diverses manières dans la traduction. Par exemple, dans le poème "Indifférence, murmure», le poète écrit que la musique va "différant chaque note jusqu'à l'absence» (de Bellefeuille, 1986, p. 15). On est tenté d'y percevoir un certain écho de la différance derridienne. On n'en trouve cependant nulle trace dans la traduction, où «différant» est rendu par «distinguishing and prolonging each note to the point of absence» (de Bellefeuille, 1992, p. 13). Puis, dans le poème «La toupie de Platon", on peut lire: «Des danses qu'on n'imagine même pas [...] tant elles diffèrent - encore là - le premier geste» (de Bellefeuille, 1986, p. 43). Cette fois, le traducteur donne une touche décidément derridienne à sa version en rendant ce segment comme suit: «Dances one can hardly imagine $[\ldots]$ so much they differ and defer - there still - the first gesture» (de Bellefeuille, 1992, p. 41). En français, le jeu sur «a» et sur «e» se poursuit dans ce même poème, entre "Le ce-sans-quoi» et «Le ce-sens-quoi» (de Bellefeuille, 1986, p. 43). Ici, Jones opte pour une solution habile où il s'agit également de changer une seule lettre, une consonne cette fois, pour faire basculer le sens: "This lack of reasons whereby...» et «this pack of reasons whereby» (de Bellefeuille, 1992, p. 41). 
Pour donner un autre exemple de mot pouvant trouver un écho dans la culture donnée, il est certain que, dans l'espace poétique québécois au moment où de Bellefeuille publie ce recueil, l'emploi du mot «réel» est chargé de signification. On n'a qu’à penser au recueil Le réel absolu de Paul-Marie Lapointe. À peine deux ans plus tard, ce mot figurera dans le titre d'un essai marquant de cette décennie, L'écologie du réel de Pierre Nepveu. Aussi, quand on lit, par exemple, dans le poème " $\mathrm{Du}$ chant, plutôt les lèvres ", le segment «ne plus être aveugle au réel» (de Bellefeuille, 1986, p. 20), on sent ce dernier mot lesté d'un poids sémantique lié à la tradition poétique québécoise. En anglais, «Not to be anymore blind to the real» (de Bellefeuille, 1992, p. 18) ne fait écho à rien: cela sert plutôt à introduire dans l'espace poétique anglo-canadien une autre manière d'agencer les mots, une autre façon de regarder la réalité. Les échos dissimulés comptent aussi parmi les problèmes auxquels doit se mesurer le traducteur. Ainsi, dans le titre même du poème "On s'écrierait: "Le réel" ", on lit, comme en filigrane, le verbe «s'écrirait» (de Bellefeuille, 1986, p. 39). La traduction ne conserve que l'en deçà du texte en donnant la version suivante: «One would write: "Reality”»(de Bellefeuille, 1992, p. 37).

À propos de la traduction du verbe «désencombrer», dans le poème «Désencombrer le monde entier» (de Bellefeuille, 1986, p. 57) - verbe que Jones rend simplement par «Clearing up»-, Stephanie Nutting écrit qu'il s'agit d'un autre exemple de ce qu'elle appelle la «stratégie transformative » à laquelle recourt le traducteur. Nutting examine la pratique traductive de Jones dans un texte intitulé «On D. G. Jones and Translating Outside», publié dans le collectif Writing Between the Lines réunissant des portraits de traducteurs canadiens-anglais sous la direction d'Agnès Whitfield. De manière générale, Nutting estime que les choix de traduction de Jones ont pour effet de resserrer le texte. Bien que ce dernier affirme pratiquer la traduction libre, écrit-elle, «son travail recèle une indéniable rigueur interne» (Nutting, 2006, p. 122)4. Le choix de recourir à un "phrasal verb» comme «clearing up » pour rendre le verbe «désencombrer»-ce qui transforme, notons-le, au moyen d'une modulation un verbe exprimant l'action de défaire une chose telle qu'un encombrement en une action positive consistant à

4. Sauf indication contraire, nous traduisons les extraits en langue anglaise cités dans le présent article. 
faire place nette - n'empêchera pas le traducteur de rendre plus loin le verbe «désordonner»- dans le poème «Le froid des avantguerres» (ibid., p. 73) - par un verbe de construction analogue au verbe français - «disrupt the shadows» (de Bellefeuille, 1992, p. 72). Autrement dit, il n'y a pas de recette toute faite, ou, comme l'écrit de Bellefeuille: «Il n'y a pas d'imitation» (1986, p. 48).

\section{La danse-transposition}

Vinay et Darbelnet ont proposé des définitions de divers procédés de traduction par lesquels certaines valeurs sémantiques sont transférées d'une langue à l'autre. L'un d'eux est appelé la transposition. Il consiste, expliquent-ils, «à remplacer une partie du discours par une autre» (Vinay et Darbelnet, 1977, p. 50). Ces déplacements sont nécessaires car le traducteur ne travaille pas sur "une langue neutre, uniquement faite de concepts» (ibid., p. 96). Dans le cas de Jones, on pourrait dire que le principe actif qui préside à son travail de traduction est une sorte de «dansetransposition» qui met en mouvement divers éléments des poèmes en langue originale.

Jones sait pratiquer, bien sûr, la transposition au sens plus strict et technique que lui donnent Vinay et Darbelnet. Par exemple, dans le poème «Le corps, nous l'avons" - titre qui, par sa mise en relief, comporte une forme de répétition, le pronom redoublant le substantif -, la citation mise en exergue, «la danse [...] est un délire à blanc» (de Bellefeuille, 1986, p. 45), est rendue en anglais de manière à opérer une permutation grammaticale des deux derniers éléments: "the dance [...] is a delirious blank» (de Bellefeuille, 1992, p. 43). Si les poèmes de Normand de Bellefeuille suscitent de par eux-mêmes une réflexion sur l'original et la copie, la «dansetransposition" par laquelle le recueil est donné à lire en anglais ne peut qu'inciter à poursuivre cette exploration, à prolonger cette réflexion. De cette manière, la deuxième partie de Catégoriques un deux et trois ayant la danse pour axe - partie qui constitue le deuxième temps du mouvement ternaire du recueil - coïncide avec la traduction, car celle-ci constitue à son tour, rappelons-le, le deuxième temps d'un autre mouvement ternaire qui se superpose au premier et qui englobe le texte, sa traduction et sa critique. 
Dans une postface à sa traduction, Jones écrit que Michel Serres, dans son ouvrage sur le livre de Tite-Live sur la fondation de Rome,

\begin{abstract}
fait remarquer que la danse débute quand on croit que les tremblements de la victime sacrificielle seront mal reçus par les dieux. Il en résulte que le meurtre proprement dit est remplacé par la représentation, l'exécution d'une danse par des acteurs professionnels. Le monde et sa représentation. La danse, des pieds, des sons, des images, des mots - ellemême un acte et une représentation - devient dans le texte de de Bellefeuille - un pas de deux contre la mort. (Jones, 1992, p. 76-77)
\end{abstract}

La traduction permet de répercuter ce monde de représentation, de transposition, dans un autre espace culturel et linguistique. Comme nous le montre sa version inventive, c'est une chose que Doug Jones a très bien comprise et qu'il réalise - au sens français du terme, faut-il le préciser.

\title{
Une traduction dédicacée
}

Jones a dédicacé sa traduction à sa femme, Monique Grandmangin, «mon Ariane au milieu du labyrinthe des tours sémantiques et de la syntaxe du français» (de Bellefeuille, 1992, n.p.). La consultation du fonds d'archives de D. G. Jones, déposé à l'Université de Sherbrooke ${ }^{5}$, permet de fait de constater que certaines ébauches de traductions de ce recueil sont annotées de la main de Grandmangin, traductrice qui a été longtemps chargée de cours en traduction à l'Université de Sherbrooke et collaboratrice à la revue Ellipse. Le fonds contient en outre une lettre de Normand de Bellefeuille à son traducteur ${ }^{6}$, dans laquelle l'auteur répond à une série de questions que Jones lui avait vraisemblablement posées dans une missive précédente. De Bellefeuille écrit qu'il fera de son mieux pour lui répondre même si ce livre lui «échappait déjà lors de son écriture, il y a maintenant près de sept ans». Dans plusieurs cas, en réponse à des demandes d'élucidation de la part de Jones, l'auteur avoue ne plus très bien se souvenir de la source précise de telle ou telle référence (à John Cage, à la toupie de Platon, etc.). Il s'engage

5. Fonds D. G. Jones, P73, 3,30 mètres de documents textuels. https://www. usherbrooke.ca/biblio/trouver/archives/archives-privees/p73-dg-jones/

6. Celle-ci est datée du 4 août sans que l'année soit précisée. Il s'agit sans doute de 1992. 
même à refaire "quelques petites recherches" sur certains de ces points, dans un mouvement de retour aux sources de la création par le créateur lui-même. De Bellefeuille approuve les quelques choix de traduction que Jones lui soumet. "Votre traduction, écritil, respecte le texte tout en ne craignant pas de prendre quelques libertés qui ne font qu'ajouter du sens à l'original.» L'auteur conclut sa lettre en remerciant Jones pour ce travail. Conscient de la bonne réputation de son traducteur-poète, il lui confie avoir «déjà rendu quelques amis envieux en leur disant que vous travailliez à cette traduction». Il dit avoir «bien hâte de vous/me... enfin nous relire...».

$\mathrm{La}$ recension critique de ce recueil en traduction souligne la nature méditative des poèmes en prose de de Bellefeuille. De Categorics one two E' three, Charlene Diehl-Jones retient le "chemin méditatif» que trace cette poésie à travers la musique, la danse et la peinture, sans oublier ce que le quotidien peut avoir d'idiot. Ces poèmes "portent sur la pensée, et le corps qui écoute, danse et observe tout en réfléchissant, sur le corps qui répond" (Diehl-Jones, 1993, n.p.). Le rôle que fait jouer de Bellefeuille à la répétition est également souligné dans ce compte rendu où la traduction de D. G. Jones est qualifiée d' «élégante» (ibid.). Cette épithète ne semble pas reposer sur une étude comparative des deux versions. On sent l'emploi de ce qualificatif surtout motivé par l'intention de louanger le travail de ce poète-traducteur sur la foi de la réputation qu'il s'était déjà acquise pour avoir notamment traduit dans la revue Ellipse les vers de maints poètes québécois.

Categorics one two $E^{\circ}$ three fait aussi partie du survol qu'effectue en 1993 Jane Koustas des titres traduits vers l'anglais au cours de l'année précédente pour la revue University of Toronto Quarterly. Elle estime que les lecteurs anglophones apprécieront la lecture de cet important poète québécois en traduction. Dans sa traduction «superbe» de ce recueil primé de poèmes en prose, «Jones capte le rythme essentiel, lequel s'appuie sur la répétition de mots davantage que sur les procédés habituels de la rime et de la symétrie, dans une suite de textes qui sont aussi une méditation sur l'art, la musique et la danse» (Koustas, 1993, p. 144). Le côté méditatif des poèmes de Normand de Bellefeuille est encore une fois souligné ici, sans doute parce que ce n'est pas le genre de poésie auquel on est habitué au Canada anglais, comme nous le faisions remarquer plus haut. 
En quatrième de couverture, le poète et critique Fred Wah écrit, à propos de ce recueil, qu'on a l'impression, à sa lecture, de se trouver au milieu du tumulte d'une circulation de mots et qu'on est là à se demander ce qui nous attend au détour. "Normand de Bellefeuille, écrit Wah, étudie la façon dont la langue ajoute des strates à notre monde, notre corps, notre désir. Avec Doug Jones, le traducteur, on doit regarder de chaque côté avant de traverser dans le prochain monde»(1992, n.p.). Le critique souligne ici habilement le rôle d'acteur-accompagnateur que joue le traducteur, lequel est passé maître dans l'art de la substitution des signes pour parer à la colère divine.

Mais se pourrait-il en fait que la substitution de signes soit précisément ce que souhaite en définitive la divinité? Dans une perspective biblique en tout cas, il s'agit d'un élément qui a rendu possible une certaine suite des choses. Dans la Genèse en effet, c'est à la suite d'une substitution de signes par Jacob - qui se couvre les mains de poils de chevreau pour imiter les mains velues d'Ésaü, porte les vêtements de son frère aîné et prétend avoir chassé comme lui le gibier - que ce dernier obtient la bénédiction de son père Isaac à la vue déclinante (Ge $27: 28)$. Mutatis mutandis, il n'est pas sûr que l'Auteur - créateur dans le monde des lettres puisse toujours reconnaître les fruits de sa création: à laquelle de ses «versions » revient donc le droit d'aînesse?

Pour revenir aux catégories de la sémiotique de Peirce et aux remarques sur la traduction d'Arno Renken, on peut dire que poètes, traducteurs et lecteurs n'ont pas fini de faire valser les objets, les signes et les interprétations. Le riche travail poétique de Normand de Bellefeuille («un texte») a ainsi donné lieu à une permutation des signes de la part du traducteur («un texte et un texte») qui sont à leur tour englobés dans une activité de lecture critique: un deux et trois.

\section{Références}

Blodgett, E. D. (2012a). Invention à cinq voix: une histoire de l'histoire littéraire au Canada. Trad. Patricia Godbout. Québec, Presses de l'Université Laval.

Blodgett, E. D. (2012b). «De la difficulté de traduire Saint-Denys Garneau en anglais». Études françaises, 48, 2, p. 111-119.

Brault, Jacques (1975). Poèmes des quatre côtés. Saint-Lambert, Le Noroît. De Bellefeuille, Normand (1986). Catégoriques un deux et trois. Trois- 
Rivières, Écrits des Forges.

De Bellefeuille, Normand (1992). Categorics one two E three. Trad. D. G. Jones, Toronto, Coach House Press.

Diehl-Jones, Charlene (1993). «The Dance of Reading». Books in Canada, juin. [http://www.booksincanada.com/article_view.asp?id=2079] (consulté le 3 mai 2015).

Godbout, Patricia (2010). «D. G. Jones, poète, comparatiste et traducteur». TTR, 22, 2, p. 23-35.

Jones, D. G. (1970). Butterfly on Rock: A Study of Themes and Images in Canadian Literature. Toronto, University of Toronto Press.

Jones, D. G. (1977). «Grounds For Translation». Ellipse, 21, p. 58-91.

Koustas, Jane (1993). «Translations ». University of Toronto Quarterly, 63, 1, p. 136-146.

Lapointe, Paul-Marie (1971). Le réel absolu:poèmes 1948-1965. Montréal, L'Hexagone.

Nepveu, Pierre (1988). L'écologie du réel: mort et naissance de la littérature québécoise contemporaine. Montréal, Boréal.

Nutting, Stephanie (2006). «On D. G. Jones and Translating Outside». In A. Whitfield, dir. Writing Between the Lines. Waterloo, Wilfrid Laurier Press, p. 107-138.

Renken, Arno (2009). "Traduction et étrangeté». Discours prononcé lors de la remise du Prix Raymond Aron. [http://www.riponne.ch/ textes/200912_AR_prixAron.htm] (consulté le 3 mai 2015).

Renken, Arno (2012). Babel heureuse. Pour lire la traduction. Paris, Van Dieren Éditeur, coll. «Par ailleurs».

Vinay, Jean-Paul et Jean Darbelnet (1977). Stylistique comparée du français et de l'anglais. Montréal, Beauchemin.

Patricia Godbout

Département des lettres et communications Faculté des lettres et sciences humaines Université de Sherbrooke Sherbrooke (Québec) J1K 2R1 CANADA patricia.godbout@usherbrooke.ca 